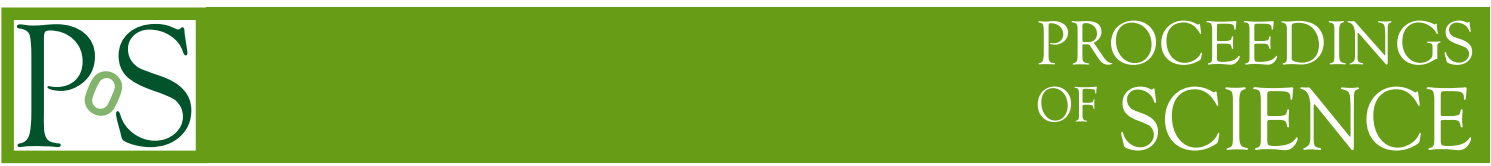

\title{
Gravitationally Lensed H I with MeerKAT
}

\author{
Roger Deane* \\ Rhodes University \\ E-mail: r.deane@ru.ac.za \\ Danail Obreschkow \\ ICRAR \\ E-mail: danail.obreschkow@gmail.com
}

\section{Ian Heywood}

University of Oxford, Rhodes University

E-mail: ian.heywoodegmail.com

The SKA era is set to revolutionize our understanding of neutral hydrogen $(\mathrm{H} \mathrm{I})$ in individual galaxies out to redshifts of $z \sim 0.8$; and in the $z>6$ intergalactic medium through the detection and imaging of cosmic reionization. Direct $\mathrm{H}$ I number density constraints will, nonetheless, remain relatively weak out to cosmic noon $(z \sim 2)$ - the epoch of peak star formation and black hole accretion - and beyond. However, as was demonstrated from the 1990s with molecular line observations, this can be overcome by utilising the natural amplification afforded by strong gravitational lensing, which results in an effective increase in integration time by the square of the total magnification $\left(\mu^{2}\right)$ for an unresolved source. Here we outline how a dedicated lensed H I survey will leverage MeerKAT's high sensitivity, frequency coverage, large instantaneous bandwidth, and high dynamic range imaging to enable a lasting legacy of high-redshift $\mathrm{H}$ I emission detections well into the SKA era. This survey will not only provide high-impact, rapid-turnaround MeerKAT science commissioning results, but also unveil Milky Way-like systems towards cosmic noon which is not possible with any other SKA precursors/pathfinders. An ambitious lensed H I survey will therefore make a significant impact from MeerKAT commissioning all the way through to the full SKA era, and provide a more complete picture of the H I history of the Universe.

MeerKAT Science: On the Pathway to the SKA,

25-27 May, 2016,

Stellenbosch, South Africa

\footnotetext{
* Speaker.
} 


\section{Introduction}

The evolution of neutral hydrogen is fundamental to our understanding of galaxy evolution. Our current cosmological picture is that following recombination at $z \sim 1100$, the Universe was predominately neutral until reionisation occurred between $z \sim 6-10$, driven by accretion onto supermassive black holes and star formation in the first galactic haloes. Followed by what the current data argue to be a relatively rapid reionoization epoch between $z \sim 6-7$ (e.g. [31]), most neutral hydrogen is now found in galaxies where it is shielded and/or replenished (e.g. [1]). However, the evolution of neutral hydrogen in $z<6$ galaxies is poorly constrained, as direct detections are only routinely possible out to $z \lesssim 0.2$. Limits from Ly- $\alpha$ absorbers suggest little to no evolution, albeit with large systematic and statistical uncertainties (e.g. [29]), while statistical techniques (i.e. stacking and intensity mapping; e.g. [16, 5]) are yet to yield robust, high precision results beyond low redshifts (e.g. [9]).

The above, data-limited picture is set to change drastically over the next 5-10 years, which will fundamentally transform our understanding of the cosmic evolution of neutral hydrogen. This will be driven by a wide range of new radio telescopes, including MeerKAT, ASKAP, SKA1-MID, as well as the upgraded VLA, WSRT APERTIF project and the GMRT. These telescopes will perform multi-tiered H I surveys (an indicative summary can be found in [2]), which will increase H I sample sizes by several orders of magnitude. While the deepest of these surveys will detect individual galaxies out to redshifts of $z \sim 1$ for the most massive $\mathrm{H}$ I systems $\left(M_{\mathrm{HI}} \gtrsim 10^{11} \mathrm{M}_{\odot}\right)$, all will have H I limiting masses well above a Milky Way-like system at $z \gtrsim 0.8$. Higher redshifts will be accessible through $\mathrm{H}$ I absorption detections which will provide important insights (e.g. [11]), however, these still suffer from significant sources of uncertainty (e.g. spin temperature, gas morphology) that limit extrapolation to the cosmic evolution of $\mathrm{H} \mathrm{I}$ in galaxies.

The greatly enhanced sensitivity, survey speed and instantaneous H I redshift coverage of these next-generation instruments will result in a dramatically increased H I-detection probability of both known and unknown lensed sources. Here we assume the canonical definition of strong lensing as a total magnification $\mu \geq 2$. This corresponds to an effective increase in integration time by a factor of $\mu^{2} \geq 4$, assuming the lensed sources are unresolved in the image plane. Since routine H I detection have so far been limited to $z \lesssim 0.2\left(D_{\mathrm{L}} \sim 980 \mathrm{Mpc}\right)$, the chance alignment of two unrelated galaxies within this volume is negligibly small. However, since the chance alignment probability goes as $r_{\max }^{6}$ for a Euclidean non-evolving Universe, where $r_{\max }$ is the distance that corresponds to the maximum redshift, the lensing probability is set to dramatically increase as H I sensitivity limits increase to $z \sim 1\left(D_{\mathrm{L}} \sim 6800 \mathrm{Mpc}\right)$. The opening of the $\mathrm{H}_{\mathrm{I}}$ Universe by these future radio facilities therefore opens an additional window through lensing and enables the detection of $\mathrm{H}$ I at even greater cosmological distances than otherwise expected.

The two highest priority MeerKAT science cases are a deep H I survey and pulsar timing (Baker et al., Bailes et al., these proceedings). However, what had not previously been considered in the MeerKAT science case, is the enhanced ability to detect H I emission in high-redshift galaxies by using the natural amplification of gravitational lensing. In this contribution, we consider the scientific opportunities this would provide and the niche that MeerKAT will occupy in this domain until the SKA-era. We discuss possible observing strategies to maximise of the scientific return of lensed $\mathrm{H}$ I detections and outline how this will enhance H I science with MeerKAT. We also outline 
how gravitational lensing will enable high-impact, rapid-turnaround early science with MeerKAT and provide a preview of the revolutionary impact the LADUMA and MIGHTEE-HI surveys will have, once completed.

\section{HI surveys in the MeerKAT and SKA era}

A primary objective of the SKA from its earliest developments was to detect Milky Way-like systems in H I emission at high redshift $(z \sim 2$; [34]). This objective has evolved into a rich science case for $\mathrm{H}$ I, covering many key aspects of galaxy evolution physics and cosmology from the detection of the epoch of reionization ([32]), to Baryonic Acoustic Oscillations (BAO) studies ([30]), and potential direct expansion measurements ([15]). Current specifications of SKA1-MID Band 1 would only enable detections above $z>0.8$ for $M_{\mathrm{HI}} \gtrsim 10^{10} \mathrm{M}_{\odot} \mathrm{H}$ I galaxies in the deep, $1 \mathrm{deg}^{2}$ survey outlined by [33] and SKA Science Book chapters referred to in their overview. They describe three fiducial SKA1-MID H I surveys that cover extragalactic fields: the medium wide, medium deep and deep surveys which cover areas of $\Omega=400,20,1 \mathrm{deg}^{2}$ respectively, the first two of which are limited to Band 2 and its corresponding $\mathrm{H}$ I redshift limit of $z<0.5$. In addition to this, there may be a 10,000 hour SKA1-MID survey, with a commensal H I cosmology and galaxy evolution design, however, the survey design is yet to be determined (see [33]).

From an SKA precursor/pathfinder perspective, the only survey capable of detecting $\mathrm{H}$ I emission beyond $z \gtrsim 0.6$ will be the dedicated, several thousand hour MeerKAT LADUMA H I survey - a single pointing $\left(0.6 \mathrm{deg}^{2}\right.$ at $\left.z=0\right)$ covering the Extended Chandra Deep Field South (ECDFS) using both L- and UHF bands (Baker et al., these proceedings). This will have strong complementarity with the spectral line component of the MIGHTEE survey, which will cover an area of $\Omega \sim 20 \mathrm{deg}^{2}$ and hence provide better constraints on the H I mass function at the high end out to a redshift of $z<0.58$ [19].

\section{Semi-analytic lensed HI simulation}

While none of the above H I surveys will be able to detect Milky Way-like galaxies at $z \sim 1$, a ma-

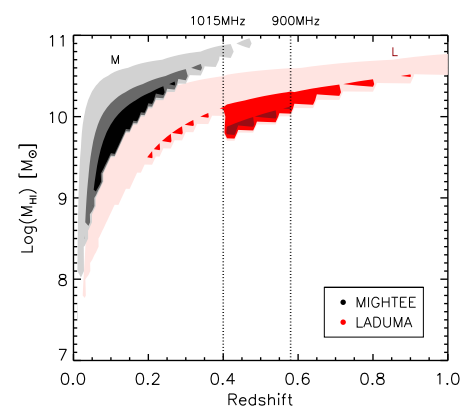

Figure 1: Directly reproduced from [19]. The coverage in the redshift- $M_{\mathrm{HI}}$ plane for LADUMA and MIGHTEE, demonstrating the lack of direct detections beyond $z \gtrsim 1$, which lensing could enable. The parameter space is divided into cells of width 0.01 in redshift and 0.1 in $\log \left(M_{\mathrm{HI}}\right)$. The contour levels for both surveys are the same, surrounding regions of parameter space containing at least one (faint shading), 10 (medium shading), and 25 (dark shading) galaxies per cell. 
jor opportunity to do so lies in the use of strong gravitational lensing. Given this opportunity, we designed a simulation (detailed in [8]) to address several questions, including: what are the surveyindependent $\mathrm{H}$ I lensing statistics as a function of redshift? How will SKA (precursor/pathfinder) surveys perform in making detections? In the absence of a pre-defined survey, which telescope is best suited to detecting lensed systems? What is the actual scientific utility of detecting lensed $\mathrm{H}$ I systems? To address these questions, we used a semi-analytic model producing realistic H I disks in terms of size, density profiles and rotation structure. This model was run on the Millennium simulation of cosmic structure, enabling us to account for realistic galaxy-galaxy clustering when computing the alignment of foreground and background galaxies. For sufficiently aligned pairs, realistic H I-lensing factors were computing using general relativistic ray tracing, applied individually to each frequency channel (see Fig. 2). Our simulation uses a $150 \mathrm{deg}^{2}$ field-of-view mock observing cone out to a redshift limit of $z_{\text {source }}<4$, enabling the prediction of detection rates of virtually all future $\mathrm{H}$ I surveys (excluding SKA-LOW), for intrinsic velocity-integrated $\mathrm{H}$ I flux densities above $0.1 \mathrm{mJy} \mathrm{km} \mathrm{s}^{-1}$.

These simulations resulted in a wide range of conclusions, two of which are particularly relevant to these proceedings. Firstly, none of the SKA pathfinder/precursor wide-field surveys will

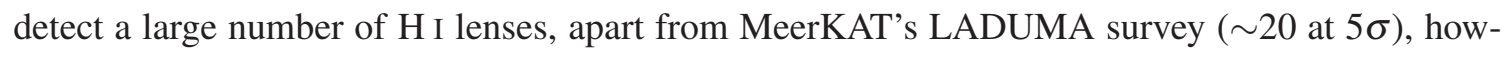
ever, the vast majority of those will have low magnification $(\mu \sim 2)$ with limited scientific utility. Secondly, MeerKAT is the best-suited instrument to make lensed H I detections pre-SKA, provided it performs a targeted survey of known gravitational lenses, as well as blind, single pointing searches towards well-modelled, high mass clusters with minimal associated diffuse radio emission. In the remainder of this contribution, we focus on MeerKAT-specific technical advantages and scientific opportunities with regards to detecting lensed $\mathrm{H}$ I systems.

\section{MeerKAT's design advantages for lensed $\mathrm{H}$ I detection}

MeerKAT's design was optimised toward its two highest priority science themes: neutral hydrogen beyond the local Universe and pulsar searching and timing. A few of the resultant telescope attributes are a 'pinched' core array configuration (50\% of the collecting area within $1 \mathrm{~km})^{1}$; excellent receiver sensitivity in the L- and UHF bands; wide field-of-view ( $\sim 2$ and $0.6 \mathrm{deg}^{2}$ for UHF and L-band respectively); wide bandwidth (435 and $770 \mathrm{MHz}$ UHF and L-band respectively); and an offset-Gregorian feed for completely unblocked aperture to maximise the imaging dynamic range. The array configuration will result in high fidelity imaging and the ability to accurately model bright sources, while still not achieving too high an angular resolution as to begin to resolve out strong lenses with their increased solid angles. However, even in the case that this does occur - the pinched core array configuration will mean that just a small fraction of the total sensitivity is lost to the highest magnification cases.

If one combines the above with the extremely radio quiet site, as well as the imaging quality and measured phase stability from MeerKAT Array Release 1 (AR1), this suggests MeerKAT is supremely placed to detect high redshift, gravitationally lensed $\mathrm{H}$ I emission. The combination of the wide FoV and large instantaneous bandwidth mean a large cosmological volume (co-moving

\footnotetext{
${ }^{1} \mathrm{~A}$ pinched core refers to an array configuration that has a significant increase in filling factor within the core, thereby achieving high brightness temperature sensitivity and pulsar search efficiency.
} 
volume of $V \sim 15 \mathrm{Gpc}^{3}$ in UHF band) is observed for every pointing. This maximises the probability of serendipitous detections in the case of blind cluster surveys and enables rich commensal science opportunities.

\section{Science opportunities enabled by gravitational lensing}

Although gravitational lensing has a long history of providing a rich set of results at other wavelengths, it is important to ask what the specific scientific utility will be in the lensed H I case. Here we describe a just a few of the wide range of science applications, all of which have significant value and will further the MeerKAT H I science priority.

\subsection{Highest redshifts}

Amongst the most spectacular results from MeerKAT is likely to be the direct detection of lensed, gas-rich $\mathrm{H}$ I galaxies at the highest redshifts achievable in the pre-SKA era $(z \sim 1.45)$. Simulations predict that LADUMA will only be able to detect a $M_{\mathrm{HI}} \sim 10^{10} \mathrm{M}_{\odot}$ galaxy out to $z \sim 1$ in its $\sim 2500$ hours single UHF band pointing (see Baker et al., these proceedings; [19]). However, for a magnification $\mu=10,25$ system, this would correspond to an effective factor of $10^{2}=100,25^{2}$ $=625$ increase in observing time. So for the latter case, a 24 hour MeerKAT observation would be the equivalent of $\sim 8 \times$ LADUMA for that particular galaxy. Clearly, this will provide the deepest cosmic view of $\mathrm{H}$ I emission in galaxies. Even this high magnification system would still be within 2-3 PSFs, assuming a $r \sim 30 \mathrm{kpc}$ disk at $z \sim 1$, which is only magnified in one direction (i.e. worst case scenario, which is highly unlikely given the expected, intrinsic H I solid angles).

The key scientific applications here are to (a) reconcile the dramatic increase in cosmic star formation rate density ([17], and references therein) with the relatively constant $\mathrm{H}$ I density evolution (e.g. [29]); and (b) obtain a high-redshift H I perspective on the dichotomy between so-called main sequence star forming galaxies which are driven by secular evolution (e.g. [6]) and the starbursts that are driven by major mergers (e.g. [10]). Given the three order of magnitude increase in the cosmic star formation rate density and the significantly higher galaxy merger rate at $z \sim 2$, direct $\mathrm{H}$ I emission measurements at the highest redshifts are of key importance and likely to only be delivered through strong gravitational lensing. Since CO transitions are readily detectable at these redshifts, lensed $\mathrm{H}$ I detections will place the highest redshift constraints of the molecularto-atomic hydrogen ratio out to $z \gtrsim 1$, a key parameter in understanding the cosmic star formation history [27].

\subsection{Low H I mass systems at cosmological distances}

As shown by our simulations in [8], two factors drive the dramatic increase in probability that lower mass H I galaxies $\left(\lesssim 10^{9} \mathrm{M}_{\odot}\right)$ are strongly lensed: (a) their increased space density, and (b) their smaller solid angles with respect to higher mass systems, which for H I disks typically results in higher magnification factors (all other factors being equal). What this implies is that MeerKAT's specifications make it extremely well suited to both targeted and serendipitous detections of lower mass $\mathrm{H}$ I systems across a wide range of redshift space $(0.2 \lesssim z \lesssim 0.6)$. Furthermore, it may even discover extremely high magnification systems $(\mu>50)$ that have intrinsically small solid angles and lie directly on a gravitational lens caustic or cusp. These possibilities open up the opportunity 
for detailed study of lower mass $\mathrm{H}$ I galaxies simply not possible with any other $\mathrm{H}$ I surveys that are currently planned.

While it is more difficult to discern high magnification, low mass galaxies from high mass, non-lensed galaxies at similar redshifts, these are in principle easily to distinguish though crossmatching with foreground, low impact factor galaxies, particularly bright ellipticals which are easily identifiable from optical/NIR imaging.

\subsection{Efficient lens selection and a unique probe of foreground dark matter haloes}

Two properties of H I lends itself to efficient gravitational lens selection, provided the appropriate survey is designed. Firstly, the redshifted H I line is very isolated in the frequency range $580 \mathrm{MHz}<v<1420 \mathrm{MHz}(1.45>z>0)$, although $\mathrm{OH}$ megamasers may prove to be a sciencerich contaminant [4]. Secondly, the high end of the H I mass function is very steep $\left(n \propto s^{-3}\right)$. This means that deep H I observations can apply a very similar lens selection technique to that performed at far-infrared/(sub)mm wavelengths in the Herschel-ATLAS survey [22], and with the South Pole Telescope, both of which had SMA/ALMA/HST followup with spectacular results [36]. The basic idea is that given the extreme rarity of the highest luminosity systems and the steep drop off in number counts, any object that deviates significantly from the statistical number density expectation is likely to be lensed. Following the removal of some obvious contaminants (blazars and Galactic objects), [22] were able to achieve an unprecedented $\sim 100$ percent lens selection rate. H I surveys could apply the same technique, however, with the huge advantage of spectral line capability (i.e. a 3D rather than $2 \mathrm{D}$ selection). This would make cross-identification easier and also open the opportunity to discover systems that have a few velocity channels that have extremely high magnification factors. In the latter case, the detection probability may be larger for a channel search width significantly smaller than the galaxy's full velocity width, as demonstrated in Fig. 2. In addition to this, MeerKAT's design and sensitivity may enable the simultaneous detection of the foreground lens in either $\mathrm{H}$ I emission and/or absorption.

What this all means is extremely high efficiency lens selection and redshift measurement, which equates to scientifically-rich samples to study foreground dark matter halo statistics. While large volume SKA H I surveys will be required to take full advantage of this opportunity, MeerKAT will undoubtedly pave the way.

\subsection{Probing the global kinematics of galaxies at high redshift}

Lensed HI-line detections will constrain the maximum velocity width $\left(V_{\max }\right)$, which, in combination with a sufficiently deep adaptive optics or space-based NIR/optical imaging, allows a measurement of the baryonic angular momentum. The larger extent of $\mathrm{H} \mathrm{I}$ in galaxies means that these lensed $\mathrm{H}$ I detections are likely to become the best baryonic angular momentum measurements at $z \sim 1$, adding an important evolutionary perspective to the science case presented in The SKA as a doorway to angular momentum [26].

\section{MeerKAT lensed HI survey strategy}

There are a range of strategies that a dedicated lensed H I survey with MeerKAT could take. Here we outline some considerations to make in formulating an observational programme that includes 


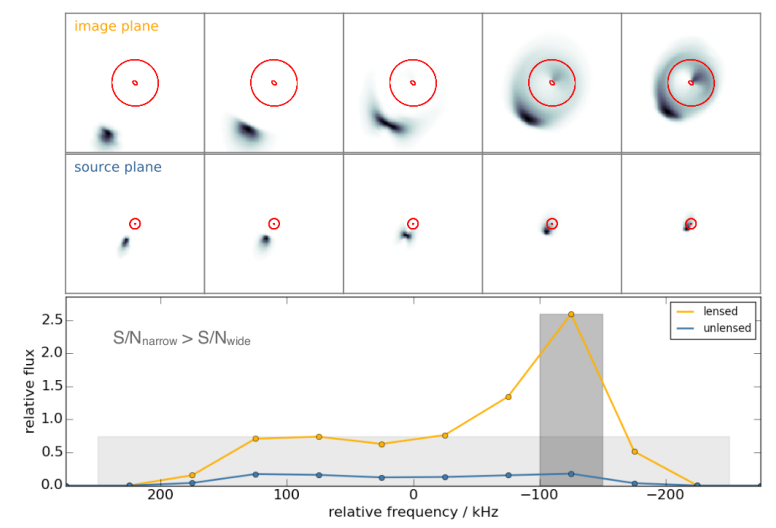

Figure 2: Reproduced from [8]. Top: Image and source plane channel maps of a Milky Way mass H I disk (200 kHz. channels), demonstrating how the approaching H I emission overlaps with the inner lens caustic and results in a full $\mathrm{H}$ I Einstein ring in some channels. Each frame is $6.4 \times 6.4 \operatorname{arcsec}^{2}$. The caustics/critical curves are plotted in red. Bottom: Lensed and unlensed H I profiles of the same system at higher frequency resolution $(50 \mathrm{kHz})$. Differential magnification is seen between channels to the extent that the probability of detection is higher for a narrow $(\sim 50 \mathrm{kHz})$ channel width, rather than the full $\mathrm{H}$ I line width.

a proof-of-concept stage, followed by dedicated targeted observations of known lenses (selected at other wavelengths); and deep, high-mass cluster observations to enable serendipitous detections.

\subsection{Proof-of-concept / science commissioning observations}

The MeerKAT Large Survey Programmes (LSPs) have been allocated $\sim 70$ percent of the observing time on MeerKAT during the $\sim 5$ years it will operate before becoming part of SKA1-MID. Many of the approved LSPs require the full array (Array Release 6) to achieve their scientific objectives (laid out in these proceedings). While the LADUMA and MIGHTEE LSPs will require pre-AR6 time to test data post-processing pipelines and familiarise survey team members with the data attributes, it's not clear that there will be any high impact, quick turnaround results from these in the pre-AR6 stage.

In contrast, a lensed $\mathrm{H}$ I detection at a redshift inaccessible by current radio interferometers such as the VLA ( $z \gtrsim 0.4)$ would provide a high impact science result with just a few tens of hours of observing time (i.e. $\lesssim 50 \mu$ Jy beam per $\sim 200 \mathrm{kHz}$ channel sensitivity at $\sim 1 \mathrm{GHz}$ ). This would simultaneously provide an excellent science commissioning target in both the bottom end of the L-band and top end of the UHF band. A handful of moderate redshift $(0.2 \lesssim z \lesssim 0.58)$ detections would play an important role in demonstrating the ability to detect $\mathrm{H} \mathrm{I}$ at cosmological distances in just a few tens of hours, while avoiding the calibration risks and data-processing overhead of a few hundred hour commissioning observation.

Regarding data volumes, there are clear advantages here. With observations of 24-48 hours, the data volume in the Measurement Set format is of the order of 72-144 TB (assuming 32K spectral line mode, 64 antennas, full polarisation), which means the full visibility dataset can be processed rather than being forced to combine subsets of the data in the image domain (a calibration risk for LADUMA, for example). During science commissioning, this data rate can be reduced by well over an order of magnitude by only processing a selected channel range set by the known 
redshift of the targeted lensed source. This demonstrates that lensed $\mathrm{H}$ I targets can indeed deliver rapid-turnaround, high impact science-commissioning results with relatively low calibration and post-processing risk and expense.

Lensed sources have been shown to be excellent science commissioning targets (or byproducts) in other observatories, most recently by Herschel and ALMA. For example, discoveries in the Herschel-ATLAS Science Demonstration Phase included the $z \sim 3$ Einstein-ring source SDP. 81 using the technique described in Sec. 5.3 [22], which was then followed up to produce the spectacular images seen in ALMA Science Verification 2014 Long Baseline Campaign. MeerKAT has a similar opportunity to use lensing to detect $\mathrm{H}$ I at unprecedented redshifts while still in its science commissioning phase, the followup study of which will undoubtedly yield rich scientific rewards.

\subsection{Targeted surveys}

Following the proof-of-concept detections, a next step would be to perform a targeted survey of a list of known lenses that are detected at other wavelengths. In the redshift range accessible to MeerKAT, the vast majority of known lenses (at present) were discovered in the SLACS survey [3], which identified composite (i.e. emission + absorption lines), but redshift-offset SDSS-spectra and performed high angular resolution HST followup to identify the largest sample of lensed sources to date (this includes 46 sources below MeerKAT's maximum H I redshift limit of 1.45). This preferentially selected extended star forming galaxies lensed by foreground elliptical galaxies. There are a number of other lenses in this redshift range, selected in a range of methods (CLASS, SQLS, COSMOS; see [35] and references therein), however, they make up the minority, at present.

The most promising lensed $\mathrm{H}$ I targets within these samples can be identified using the (large scatter) $M_{\mathrm{HI}}-M_{\star}$ relation of [18]. The combination of targets can be optimised based on redshift space, selection technique and detection probability. In addition there will be a significant increase in the number of lenses discovered in this redshift range in the period 2017-2023 from various multi-wavelength surveys, a HI-relevant summary of which is presented in [21].

\subsection{Blind cluster surveys}

What is perhaps the most exciting and discovery-laden aspect of a potential $\mathrm{H}$ I lensing programme, are deep observations of high mass clusters. The high projected mass surface density of a $z \gtrsim 0.1$ cluster dramatically increases the probability of lensed H I detections. The Einstein radii of the highest mass clusters are all below $\theta_{\text {Ein }} \lesssim 2$ arcmin, so well within the MeerKAT FoV. The strategy would be to target $z \sim 0.3$ clusters with accurate mass models available (from optical weak/strong lensing and X-ray analyses), such as those in the Hubble Frontier Fields ${ }^{2}$. As discussed in Sec. 4, a deep integration in the UHF band would open up a lensed H I discovery space across a comoving cosmological volume of $\sim 15 \mathrm{Gpc}^{3}$. An additional consideration would be to select clusters with minimal extended emission and hence more accurate sky models and resultant image fidelity, however, with 2016 baselines (for 64 antennas), modelling any complex source structure is a wellconstrained problem and can be further circumvented by excluded the shortest baselines in the complex gain solution determination.

\footnotetext{
${ }^{2}$ See https://archive.stsci.edu/prepds/frontier/lensmodels for a detailed comparison of five different methods for six Hubble Frontier Field clusters.
} 


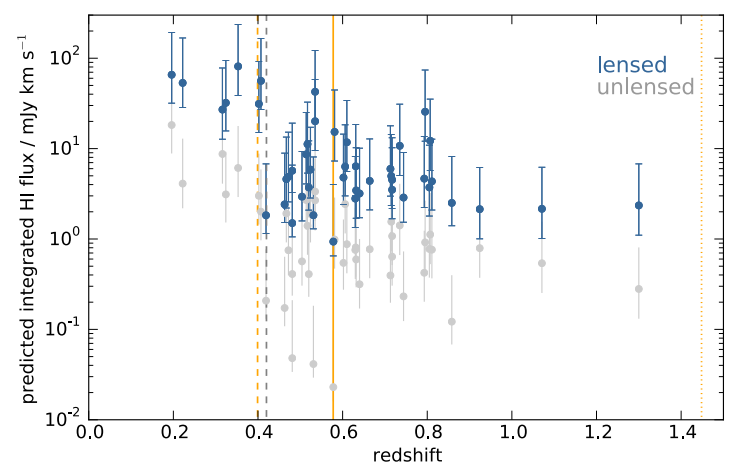

Figure 3: Illustration of possible lensing targets from just one optical survey (SLACS) where all lens models were derived from HST imaging [23]. Based on the optical magnifications, we plot both the predicted lensed (blue) and unlensed (grey) velocity-integrated $\mathrm{H}$ I flux density. Reasonable assumptions about the velocity widths leads to a high probability of detections for many systems at the $\sigma \lesssim 50 \mu \mathrm{Jy} \mathrm{beam}^{-1}$ per $200 \mathrm{kHz}$ channel sensitivity level (i.e. a few tens of hours of MeerKAT observing time). The dashed grey line indicates the maximum $\mathrm{HI}$ redshift accessible by L-band receivers on current radio interferometers (e.g. VLA and GMRT). The orange dashed and solid lines indicate the MeerKAT UHF and L-band minimum and maximum redshift coverage respectively. The dotted orange line indicates the maximum $\mathrm{H}$ I redshift accessible by the MeerKAT UHF band.

The cluster-lens searches could be carried out in two tiers: several clusters observed in both L- and UHF band for 20-50 hours; and then the ultra-deep observation of 1-2 clusters in UHF for 100-250 hours. Based on simulations from [8], the latter will result in 1 guaranteed discovery beyond $z \sim 1$, as well as several lower redshift and known lenses from optical searches. Although these data will be confusion-limited in total intensity, they will be deepest polarized view of galaxy clusters. With MeerKAT's optimised imaging fidelity, dynamic range and brightness temperature sensitivity, this could be a revolutionary project in itself with a lasting legacy into the SKA era.

\section{Complementarity with and enhancement of MeerKAT Large Survey Projects}

This contribution has focused on presenting the lensed H I case with MeerKAT. For this reason, as well as brevity, we have not spent much time on the revolutionary impact that the MeerKAT LADUMA and MIGHTEE-HI surveys will have on our view and understanding of the H I Universe. However, by carrying out an ambitious lensing programme, one can greatly enhance these surveys and almost consider $\mathrm{H}$ I lensing as a third tier in the MIGHTEE-HI and LADUMA LSPs.

As discussed in Sec. 6.2, gravitational lensing enables the detection of systems of mass and at redshifts not directly accessible by LADUMA. This will not only add to the output of LADUMA, but also provide a very useful cross-check with stacking, provided a sufficient number of lensed H I detections are made at high redshift. Therefore, from a high-redshift $\mathrm{H}$ I risk perspective, lensing circumvents most of the calibration risks that the deep LSPs will face and will return results on very short timescales.

\section{Synergy with other major facilities and surveys}

We expect this lensed H I programme to have strong synergies with other observatories and large- 
scale multi-wavelength imaging and spectroscopic surveys. This will be from both a target selection and detection follow up perspective. Over the course of the next $\sim 5$ years, significantly more lenses will be discovered/confirmed in the wide area, optical/NIR imaging and spectroscopic programmes (see [21] for a summary), so the targeted lensed H I component to this proposed programme would not be target limited. In the follow up context, there are a slew of multi-wavelength observatories that enable lensed H I sources to each become individual cosmic laboratories in their own right, including ALMA and JWST.

\section{Pathway to and legacy value in the SKA era}

Following from MeerKAT, the SKA1-MID array will take lensed H I studies forward in two primary ways. Firstly, the SKA1-MID factor $\sim 3$ sensitivity increase and extended Band 1 redshift coverage to $z=3$ will allow blind surveys to discover large samples of lensed H I sources and not be limited to massive cluster searches. This will fully exploit the selection technique described in Sec. 5.3. Secondly, for the most massive galaxy clusters $\left(\theta_{\text {Ein }} \gtrsim 20 \operatorname{arcsec}\right)$, it will become possible to spatially resolve intermediate redshift sources. A dedicated MeerKAT H I lensing programme will provide immediate targets for SKA1-MID as well as guidance for optimised SKA cluster and blind lensed H I surveys. In the full SKA era, lensed H I may well be the most efficient lens selection tool, particularly when used in combination with the lensed continuum emission, H I absorption/emission in the foreground lens, and $\mathrm{OH}$ lines in the same band (see [20]).

\section{Conclusion}

The revolutionary role MeerKAT has been designed to play in uncovering the $\mathrm{H}$ I history of the Universe will be dramatically enhanced by exploiting the natural magnification afforded by strong gravitational lensing. In these proceedings, and in [8], we have made the case for the following statements, (i) MeerKAT will (emphatically) be the best facility to detect lensed H I pre-SKA; (ii) a dedicated MeerKAT H I lensing programme will provide high-impact, rapid-turnaround early science and will make the highest redshift detections of $\mathrm{H}$ I emission in galaxies pre-SKA; (iii) direct lensed $\mathrm{H}$ I detections at high-redshift will provide important cross-checks with stacking/statistical methods, provided a sufficient number of lensed-H I detections are made; (iv) the lensed H I observing requirements pose significantly lower risk regarding calibration for high-z $\mathrm{H}$ I inference, when compared to the LSPs; (v) as has been seen in the case of sub/mm-discovered lenses, MeerKATdiscovered $\mathrm{H}$ I lenses are likely to have high legacy value well into SKA era.

\section{Acknowledgements}

We thank Natasha Maddox for allowing us to reproduce Figure 1 here and for helpful discussions.

\section{References}

[1] Barkana, R., \& Loeb, A. 2001, Physical Reports, 349, 125

[2] Blyth, S., et al. 2015, Advancing Astrophysics with the Square Kilometre Array (AASKA14), 128 
[3] Bolton, A. S., Burles, S., Koopmans, L. V. E., Treu, T., \& Moustakas, L. A. 2006, ApJ, 638, 703

[4] Briggs, F. H. 1998, A\&A, 336, 815

[5] Chang, T.-C., Pen, U.-L., Bandura, K., \& Peterson, J. B. 2010, Nature, 466, 463

[6] Daddi, E., et al. 2007, ApJ, 670, 156

[7] Deane, R. P., Heywood, I., Rawlings, S., \& Marshall, P. J. 2013, MNRAS, 434, 23

[8] Deane, R. P., Obreschkow, D., \& Heywood, I. 2015, MNRAS, 452, L49

[9] Delhaize, J., Meyer, M. J., Staveley-Smith, L., \& Boyle, B. J. 2013, MNRAS, 433, 1398

[10] Di Matteo, P., Combes, F., Melchior, A.-L., \& Semelin, B. 2007, A\&A, 468, 61

[11] Gupta, N., Salter, C. J., Saikia, D. J., Ghosh, T., \& Jeyakumar, S. 2006, MNRAS, 373, 972

[12] Hodge, J. A., et al. 2012, ApJ, 760, 11

[13] Johnson, T. L., et al. 2014, ApJ, 797, 48

[14] Jonas, J. L. 2009, IEEE Proceedings, 97, 1522

[15] Kloeckner, H. R., et al. 2015, Advancing Astrophysics with the Square Kilometre Array, 27

[16] Lah, P., et al. 2007, MNRAS, 376, 1357

[17] Madau, P., \& Dickinson, M. 2014, ARA\&A, 52, 415

[18] Maddox, N., Hess, K. M., Obreschkow, D., Jarvis, M. J., \& Blyth, S.-L. 2015, MNRAS, 447, 1610

[19] Maddox, N., Jarvis, M. J., \& Oosterloo, T. A. 2016, MNRAS, 460, 3419

[20] McKean, J., et al. 2015, Advancing Astrophysics with the Square Kilometre Array (AASKA14), 84

[21] Meyer, M., et al. 2015, Advancing Astrophysics with the Square Kilometre Array (AASKA14), 131

[22] Negrello, M., et al. 2010, Science, 330, 800

[23] Newton, E. R., et al. 2011, ApJ, 734, 104

[24] Obreschkow, D., Croton, D., De Lucia, G., Khochfar, S., \& Rawlings, S. 2009, ApJ, 698, 1467

[25] Obreschkow, D., Klöckner, H.-R., Heywood, I., Levrier, F., \& Rawlings, S. 2009, ApJ, 703, 1890

[26] Obreschkow, D., et al. 2015, Advancing Astrophysics with the Square Kilometre Array, 138

[27] Obreschkow, D., \& Rawlings, S. 2009, ApJL, 696, L129

[28] Oguri, M., \& Blandford, R. D. 2009, MNRAS, 392, 930

[29] Prochaska, J. X., \& Wolfe, A. M. 2009, ApJ, 696, 1543

[30] Rawlings, S., et al. 2004, New Ast. Reviews, 48, 1013

[31] Robertson, B. E., et al. 2013, ApJ, 768, 71

[32] Santos, M., et al. 2015, Advancing Astrophysics with the Square Kilometre Array (AASKA14), 19

[33] Staveley-Smith, L., \& Oosterloo, T. 2015, Advancing Astrophysics with the SKA (AASKA14), 167

[34] Taylor, A. R., \& Braun, R. 1999, Science with the SKA : a Next Generation World Radio Observatory,

[35] Treu, T. 2010, ARA\&A, 48, 87

[36] Vieira, J. D., et al. 2013, Nature, 495, 344 